\title{
Stem cell therapies for neuropathic pain
}

\author{
JASON SEEWOODHARY, JOHN N HARVEY
}

\begin{abstract}
Neuropathic pain is a large-scale epidemiological problem affecting $13-26 \%$ of the diabetic population. The complex aetiology and pathophysiology coupled with the lack of a diagnostic test for the underlying cause renders the assessment of neuropathic pain subjective and the treatment difficult, especially as current licensed treatments are limited in their application towards the attainment of palliation.

Cell therapies offer a novel curative therapeutic dimension for neuropathic pain. This is based on replacing damaged neuronal tissue, protecting against progressive nerve damage, and releasing soluble factors that act in a paracrine or endocrine manner, which facilitate repair and reversal of the pathology that underlies the genesis and propagation of damage within the somatosensory system. Cell therapies with potential utility for the treatment of neuropathic pain include embryonic stem cells, adult stem cells and induced pluripotent stem cells.
\end{abstract}

Key words: stem cell, neuropathic pain, cell therapy, regeneration, analgesia, diabetes

\section{Introduction}

Current licensed therapies for the treatment of neuropathic pain are limited in their application towards the attainment of palliation. They are not disease-modifying or neuroprotective, being incapable of reversing or repairing the pathology that underlies the genesis and propagation of damage within the somatosensory system. Stem cells may offer a novel curative regenerative therapeutic dimension. This review will discuss the potential utility of stem cells for the treatment of neuropathic pain.

\section{Neuropathic pain}

Neuropathic pain is a subtype of pain defined by IASP as "pain

Department of Diabetes Mellitus and Endocrinology, Wrexham Maelor Hospital, Wrexham, UK

Address for correspondence: Dr Jason Seewoodhary Department of Diabetes Mellitus and Endocrinology, Wrexham Maelor Hospital, Croesnewydd Road, Wrexham, LL13 7TD, UK.

Tel: +44 (0)7908 070455

E-mail: seewoodharyj@hotmail.com

Br J Diabetes Vasc Dis 2014;14:2-9

http://dx.doi.org/10.15277/bjdvd.2014.002

\author{
Abbreviations and acronyms \\ BDNF brain derived neurotrophic factor \\ ES embryonic stem \\ GABA gamma amino butyric acid \\ GAD-1 glutamate decarboxylase-1 gene \\ GAD-65 glutamate decarboxylase- 65 gene \\ $\mathrm{GAL}$ galanin \\ GFP green fluorescent protein \\ GFP-NSC green fluorescent protein labelled neural stem cell \\ hMSC human mesenchymal stem cell \\ HSV herpes simplex virus \\ IASP International Association for the Study of Pain \\ IL interleukin \\ iPS induced pluripotent stem \\ MGE medial ganglionic eminence \\ mRNA messenger ribonucleic acid \\ miRNA micro ribonucleic acid \\ MSC mesenchymal stem cell \\ NGF nerve growth factor \\ NMDA N-methyl-D-aspartate \\ NSC neural stem cell \\ piPSC protein induced pluripotent stem cell \\ SVZ subventricular zone
}

arising as a direct consequence of a lesion or disease affecting the somatosensory system". The predominant symptom is paroxysmal episodes of stabbing or shooting pain arising in an area of hyperexcitability or numbness. Other symptoms include: spontaneous pain, hyperalgesia and allodynia; dyaesthesias; abnormal thermal sensations; and deep seated gnawing pain.

\section{Epidemiology}

Neuropathic pain is a large-scale problem; epidemiological data estimate neuropathic pain affects $13-26 \%$ of diabetic patients. ${ }^{1}$ However, due to the complex aetiology of neuropathic pain coupled with the lack of a diagnostic test and standardised measurement methods, exact data are deficient, which renders the overall prevalence difficult to quantify. Accordingly the health economic costs of neuropathic pain to society are undetermined.

\section{Aetiology and pathophysiology}

The aetiology of neuropathic pain can be categorised morphologically into four groups: peripheral nervous system and multifocal lesions e.g. diabetic mononeuropathy; peripheral nervous system generalised polyneuropathies e.g. alcohol related neuropathy; central nervous system lesions e.g. spinal cord injury; and complex neuropathic disorders, such as complex regional pain syndrome types I and II. ${ }^{2}$ However, this classification system is not universally accepted. 
Figure 1. An overview summary of the peripheral and sensory mechanisms leading to neuropathic pain.

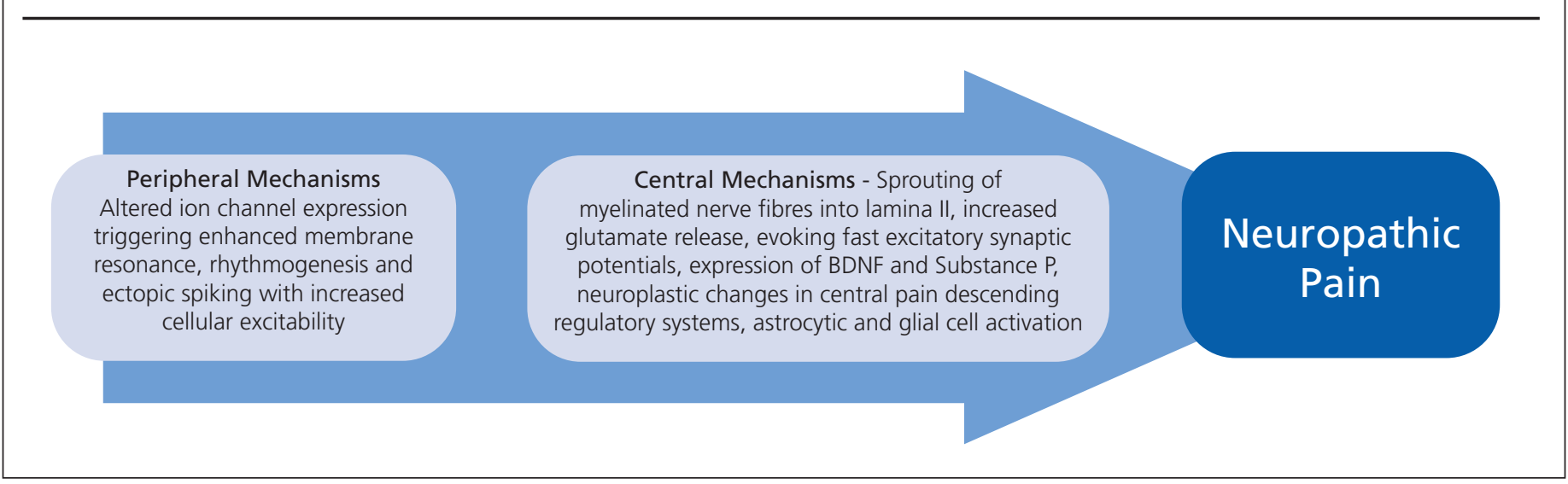

The pathophysiology of neuropathic pain is complex and results from several processes, which cumulatively lead to peripheral and central sensitisation associated with ectopic activity and hyperexcitability in pain pathways. ${ }^{3}$ This is associated with histopathological changes within affected nerves characterised by: Wallerian degeneration; sprouting; formation of end-neuromas and neuromas-in-continuity; and compression induced atrophy. ${ }^{4}$ Peripheral mechanisms act on nociceptors. The hyperactivity in nociceptors induces hyperexcitability in the spinal cord and brain, referred to as central sensitisation. A schematic depicting the various alterations in nerve function leading to neuropathic pain is illustrated in Figure 1.

\section{Diagnosis and treatment}

Neuropathic pain is diagnosed clinically. Screening methods are based on questionnaires such as the Leeds Assessment of Neuropathic Symptoms and Signs or the pain DETECT questionnaire.

The Neuropathic Pain Special Interest Group of the IASP has developed evidence-based guidelines for the pharmacological treatment of neuropathic pain. ${ }^{5}$ Based on the results of randomised controlled trials, first-line agents include: tricyclic antidepressants, selective serotonin and noradrenaline reuptake inhibitors, voltage-gated $\mathrm{Ca}^{2+}$ channel $\alpha 2-\delta$ ligands, and topical local anaesthetics. Second-line medicines include opioid analgesics and tramadol. Third-line drugs include valproate, topiramate, mexiletine, and topical capsaicin. ${ }^{6}$

Non-pharmacological treatments include: surgical and chemical sympathectomy, which are limited in their application by a sparse evidence base and considerable complications; 7 neurodestructive procedures, which are hindered by a risk of exacerbating symptomatology; neurostimulation e.g. transcutaneous electrical nerve stimulation, although this is not based on conclusive evidence; 8 spinal cord stimulation; acupuncture; and cognitive behavioural therapy.

\section{Limitations of current treatment}

Therefore, the management of neuropathic pain is challenging with a poor prognosis. Treatment is hindered for a number of reasons: there is no definitive corroboration between published guidelines regarding the assessment of first-, second-, and thirdline pharmacotherapy; the evidence underlying published algorithms is biased in favour of peripheral neuropathic pain disorders as opposed to central neuropathic pain; evidence from trials is biased in favour of monotherapy over combination therapy; guidelines and algorithms are sourced from appraisals of independent heterogeneous controlled trials rather than headto-head comparative studies; there is a high number of negative clinical trials with equivocal data; and the short duration of most trials provides limited data on chronic neuropathic pain. Additionally, the utility of current licensed drugs is further hindered by dose-limiting side-effects. Emerging evidence suggests the multifactorial challenges associated with the treatment of neuropathic pain may be surmountable by regenerative approaches based on the utility of cell therapies.

\section{Stem cells}

Stem cells are undifferentiated cells capable of unlimited proliferation and self-renewal whilst retaining the potential towards differentiation into any cell type of endodermal, ectodermal or mesodermal origin. There are three main types of stem cells: ES cells, adult stem cells, and iPS cells.

The main advantages of stem cells hone in on their potential use for regenerative therapies, with the overall aim of repairing or replacing diseased tissues and organs. Stem cell technology provides a potentially limitless purified population of patient- and disease-specific cells, which confers a range of clinical benefits. These include: understanding the pathogenesis of disease; facilitating drug discovery; and generating cells for transplantation.

\section{Embryonic stem (ES) cells}

ES cells are pluripotent cells derived from the inner cell mass of the developing blastocyst. ${ }^{9}$ ES cells confer the advantage of being: renewable; accessible to genetic modifications; and expandable in vitro for lengthy periods. Thus ES cells can be yielded in very high purified quantities for potential regenerative purposes. Disadvantages of ES cells include: a relatively high tumorigenic potential; transplant rejection; and ethical concerns relating to disaggregating the developing blastocyst. ${ }^{10}$ 


\section{Adult stem cells}

Adult stem cells are multipotent undifferentiated cells. They are derived from specific tissues within the embryo, foetus or adult e.g. the SVZ situated throughout the lateral walls of the lateral ventricles, which contains NSCs, or the bone marrow, which contains two types of adult stem cells, namely, MSCs and haematopoietic stem cells. The amniotic membrane is also a plentiful source of non-immunogenic MSCs, which are easily and non-invasively harvested. Amniotic membrane MSCs have demonstrable anti-inflammatory properties, which have been used clinically in pain relief and wound healing..$^{11,12}$

Advantages of adult stem cells include: self-renewability; fewer ethical issues relative to ES cells; and the potential to be harvested from easily accessible organs and expanded. Furthermore, adult stem cells have a superior safety profile with a lower tumorigenic potential relative to ES cells. Disadvantages include: a lower degree of plasticity, expandability, and renewability, coupled with a greater susceptibility to senescence compared to ES cells; and invasive harvesting methods e.g. bone marrow trephine and biopsy to obtain MSCs. Furthermore, in contrast to ES cells, adult stem cells are rarer in number in mature tissues. This is significant as large numbers of cells are needed for stem cell replacement therapies.

\section{Induced pluripotent stem (iPS) cells}

iPS cells are derived from non-pluripotent somatic cells such as dermal fibroblasts, which have been transformed and genetically 'reprogrammed' into a pluripotent state akin to ES cells. This is achieved by transfection with transcription factors such as Oct3/4, Sox 2 and Nanog, which are core transcription factors that repress the expression profile of differentiated cells and activate an array of genes involved in pluripotency. ${ }^{13}$ Other key transcription factors include KIf-4, Lin28 and c-Myc. Similarities to ES cells include: the expression of certain stem cell genes and proteins; viable chimera formation; chromatin methylation patterns; doubling times; teratoma formation; embryoid body formation; and potency and differentiability. ${ }^{14}$

Four traditional strategies are available to reprogramme somatic cells to an iPS cell state: viral transduction; nuclear transfer; cell fusion; and cell explantation. Reprogramming is commonly achieved with viral vectors, which can be either integrating e.g. retroviral or lentiviral vectors, or non-integrating such as adenoviral vectors.

Limitations of the transcription factor approach to make iPS cells include: a low throughput; mutations being inserted into the target cells genome; tumours, especially with c-Myc; and incomplete reprogramming. These limitations can be overcome by novel techniques to make iPS cells, which include: ES cell specific miRNA to prompt iPS cell reprogramming; ${ }^{15}$ using biomimicry with recombinant proteins injected into cells via polyarginine anchors, which has coined the nomenclature 'piPSCs' - protein-induced pluripotent stem cells; ${ }^{16}$ and small compound mimicking, which raises reprogramming efficiency. ${ }^{17}$

iPS cells offer the advantage of being: easily and non-invasively harvested; useful tools for drug development; models for disease processes in vitro; and a source of autologous cells for transplantation due to a lower risk of immunorejection. Disadvantages include a propensity towards tumorigenesis and a lack of long-term data on stability and safety. ${ }^{18}$

\section{Peripheral and central injury models}

Progress on the utility of cell based therapies for neuropathic pain research is dependent upon the application of appropriate experimental animal models of peripheral and central nerve lesions.

There are two broad groups of experimental animal models of neuropathic pain: those that localise the lesion e.g. dorsal root ganglion lesion, peripheral nerve lesion, spinal cord lesion, and dorsal and ventral root lesion; and those that describe the type of lesion e.g. transection, tumour cell invasion or laser radiation, cryoneurolysis, crush, stimulation of perineuronal inflammation, and tight or loose ligature. ${ }^{19}$ However, no single animal model entirely recaptures the full range of neuropathic pain mechanisms.

In animal models the assessment and quantification of neuropathic pain by direct evaluation is not feasible. Rather subjectively, most data obtained using animal models have relied on the use of evoked pain-related behaviours such as withdrawal responses as surrogate markers for neuropathic pain. ${ }^{20}$ Leading on from this, complexities regarding assessing neuropathic pain in animal models are further exemplified by attempts to extrapolate and identify relevant markers for spontaneous pain. This is particularly problematic for patients with neuropathic pain but rather difficult to measure in rodents. Surrogate indicators include: changes in general innate behaviours such as locomotion, burrowing, digging, excessive grooming and nesting; ${ }^{21}$ and more complicated paradigms using Pavlovian conditioning methods such as conditioned place preference and aversion. ${ }^{22}$ Notwithstanding these methods, laboratory tools for objectively assessing neuropathic pain in animal models are available and utilise two surrogate markers, namely: thermal hyperalgesia using the acetone test; and mechano-allodynia using the von Frey test. ${ }^{19}$

\section{Evidence on the utility of ES cells for the treatment of neuropathic pain}

ES cells have been used to treat neuropathic pain by regenerating GABAergic interneurons with restoration of the inhibitory tone in the dorsal horn of the spinal cord - the lack of which would otherwise contribute to the hyperexcitability that underlies allodynia and hyperalgesia. Evidence in support of this used mouse ES cell-derived MGE cortical inhibitory precursor cells, which were transplanted into a mouse model of peripheral sciatic nerve injury. ${ }^{23}$ Using ES cell-derived MGE cells that expressed GFP under the control of the Gad1 promoter, it was demonstrated that the ES cell-derived MGE grafts adapted and thrived in the novel spinal cord environment and migrated throughout the ipsilateral dorsal and ventral horns. Within two weeks of transplantation the grafted cells showed immunocytochemical evidence of differentiation towards a neuronal phenotype (NeuN+) and likewise demonstrated immunoreactivity for markers of cortical GABAergic interneurons, namely GABA, neuropeptide $Y$, parvalbumin and somatostatin. Furthermore, the transplants structurally integrated into host spinal cord circuitry as 
evidenced by neurite outgrowth, sprouting, path-finding and synapse formation with host primary afferent and post-synaptic neurons.

The grafts targeted and influenced a range of spinal cord neurons including projection neurons of lamina I that normally receive nociceptive stimuli. There was a close temporal relationship between the improvement in mechanical allodynia and integration of the grafted cells, which indicated the latter was instrumental for recovery. There was no correlation between the number of transplanted cells and observed anti-nociceptive effects, which suggests there may be a threshold above which the number of grafted cells is of less significance in attaining analgesia. There was a differential effect observed for ES cell-derived MGE transplants with efficacy demonstrated specifically for neuropathic pain, which was not matched in a model of inflammatory pain in response to formalin-induced tissue injury. This suggests MGE grafts are disease rather than symptom modifying.

The strength of Braz et al's study is based on the novel observation that ES cell-derived MGE grafts restored anti-nociceptive inhibitory GABAergic neurotransmission by structural integration into host spinal cord neuronal circuits. This contrasts with previous simplistic mechanisms of achieving the same by studies that merely focussed on releasing GABA using adenoviral and HSV vectors expressing the GABA synthesising enzyme GAD65 in neuropathic models of trigeminal neuralgia ${ }^{24}$ and spinal nerve ligation. ${ }^{25}$ Leading on from this, the conclusion of Braz et al's study has been corroborated by other groups, which increases its reliability. ${ }^{26}$

Braz et al's study failed to account for the anti-nociceptive summative effects of other co-existent endogenous inhibitory pathways, which may have confounded the cause and effect relationship between the independent and dependent variables. It was presumed the anti-nociceptive effects observed post ES cell-derived MGE transplantation was GABA mediated, as evidenced by normalisation of GAD65 mRNA levels in the peripheral nerve injury model, which is normally associated with low GAD65 mRNA levels. However, the inhibitory neurotransmitters glycine and serotonin, which co-exist in some spinal cord GABAergic neurons, may have accounted for the inhibition and anti-allodynic effects recorded. ${ }^{27}$ Additionally, evidence suggests that following nerve injury, activation of microglia results in a BDNF-mediated shift in the chloride gradient of projection neurons in lamina I and deep in the dorsal horn, which leads to GABAergic inputs becoming somewhat paradoxically excitatory and pro-nociceptive. ${ }^{28,29}$ The results from Mackie, De Koninck and Price's studies do not corroborate the functional integrative mechanism postulated by Braz et al's study.

The findings of Braz et al's study are further limited in their long-term application; they only provide data on the utility of ES cell-derived MGE grafts for the treatment of neuropathic pain for 28 days post-transplantation. Accordingly the study was unable to determine whether: the anti-nociceptive effects observed were sustained; if tolerance developed; and if the experimental models showed delayed anti-allodynic effects or developed an analgesic phenotype characterised by mechanical thresholds greater than the baseline.

A separate study used a modified retinoic acid protocol to induce differentiation of mouse ES cells into neural and glial precursors. These were used for transplantation into a mouse model of central neuropathic pain induced secondary to excitotoxic spinal cord injury. ${ }^{30}$ Following transplantation a significant attenuation in mechanical and thermal allodynia with associated paininduced behaviour, marked by excessive grooming, was observed and reduced to pre-injury levels.

In contrast to Braz et al's study, which only assessed outcome measures for 28 days post-transplantation, Hendricks et al's study observed a sustained anti-nociceptive effect for up to 60 days post-transplantation with immunohistochemical evidence of sustained graft viability. Furthermore, in contrast to Braz et al's study, ES cell-derived neural and glial grafts exhibited, in addition to anti-neuropathic effects, significant analgesic effects in a formalin-induced inflammatory hyperalgesia model suggesting they may be symptom rather than disease specific.

In a follow-up study Hendricks et al's group in 2012 anecdotally reported that predifferentiated ES cell grafts rescued a neuropathic phenotype in a mouse model. The ES cell-derived grafts exerted neuro-modulatory effects characterised by an increase in neurotrophic factors and CAMP and decreased levels of proinflammatory cytokines. This contrasts with the functional integrative mechanism in the Braz et al study.

The discrepancies between Braz et al's study and Hendricks et al's study may, in part, be explained by the different models of neuropathic pain used; Braz et al used a peripheral model of neuropathic pain (sciatic nerve injury) whereas Hendricks et al used a central model.

A separate study reported that engraftment of predifferentiated ES cells which tonically secrete serotonin and BDNF into the lumbar region of rodent models of central neuropathic pain significantly reduced mechanical allodynia and thermal hyperalgesia for up to 4 weeks post-transplantation. ${ }^{31}$ The anti-nociceptive effects of serotonin were augmented by the administration of the serotonin antagonist methysergide and the serotonin re-uptake inhibitor fluvoxamine. In contrast to Braz and Hendricks et al's studies, in this case the putative anti-nociceptive mechanism was the regeneration of interrupted descending inhibitory serotonin neuronal inputs.

In summary, the evidence on the utility of ES cells for the treatment of neuropathic pain is in its infancy. Preclinical research has focussed on the role of ES cells in restoring the inhibitory effects of GABAergic and serotonergic neurotransmission and, to a lesser extent, modulation of the hostile pro-inflammatory environment in central neuropathic pain. The utility of ES cells to regenerate or modulate other pathophysiological mechanisms of neuropathic pain such as glutamate release, C-fibre hyperexcitability, altered ion channel and NMDA receptor expression, and astrocytic and glial cell activation remains unexplored. Further research is required to determine the potential utility of ES cells to treat neuropathic pain. 


\section{Evidence on the utility of adult stem cells for the treatment of neuropathic pain}

There is a larger evidence base for the efficacy of adult stem cells for the treatment of neuropathic pain relative to ES cells. Evidence using an adult NSC line, sourced from the rodent SVZ, showed that NSCs attenuated neuropathic pain and promoted nerve regeneration in a rodent chronic constriction injury model. ${ }^{32}$ NSCs were administered via intravenous injection and preferentially homed towards the ipsilaterally lesioned nerve; evidence suggests this pattern of homing may be related to myelin modifications induced by nerve injury. ${ }^{33}$ Analgesic effects measured by a reduction in mechanical allodynia and thermal hyperalgesia were observed within three days following NSC administration, which correlated with histological evidence of NSC presence at the nerve injury site. The persistence of the analgesic effect between 7-14 days following grafting correlated with perilesional migration of a high density of fibroblasts, Schwann cells and macrophages, which facilitated regeneration, neurite outgrowth, sprouting, and an improvement in nerve morphology. Evidence suggests this is due to grafted NSCS exhibiting trophic and reparative effects. ${ }^{34}$ In support of this, the correlation between NSC administration and anti-nociceptive effects were associated with: a rapid decrease in Fos expression in laminae I-VI - high levels are normally associated with neuronal activity following noxious stimulation; ${ }^{35}$ a decrease in immunoreactivity for substance $P$ in the same region - substance $P$ has been associated with increased neuropathic pain in rodents; ${ }^{36}$ and a reduction in mRNA levels of the proinflammatory pro-algesic cytokines IL-1 and IL-6, coupled with a rise in mRNA levels of the anti-inflammatory cytokine IL-10. These findings suggest that NSCS act as local modifying agents transforming the hostile proinflammatory neurochemical environment associated with nerve injury into a more permissive milieu. This facilitates nerve regeneration and analgesia. However, the proinflammatory microenvironment may not be entirely harmful to regeneration (see later).

The strength of Franchi et al's study is based on new first evidence that intravenous administration of NSCs has bidirectional effects on the immune response - decreasing the injurious proinflammatory cytokine cascade and activating the neuroprotective anti-inflammatory cytokine response. Interestingly, the analgesic effect of NSCs preceded the morphological signs of nerve repair and was sustained after NSCs disappeared from the lesion site.

Franchi et al's findings are corroborated by a study that found MSCs transplanted into neuronal tissue ameliorated peripheral neuropathic pain. ${ }^{37}$ Similar to the immunomodulatory mechanisms from Franchi et al's study, in this case the recorded antinociceptive effects were secondary to the prevention of injury-induced changes in galanin, neuropeptide $Y$, and neuropeptide Y Y1-receptor expression in a single ligature nerve constriction rodent model. This may be explained by the bi-directional effects of galanin on neuropathic pain. Galanin is upregulated following nerve injury; however, the functional significance of this is dependent on the type and location of the GAL receptor stimulated. This may result in either: pro-nociceptive effects via activation of pre-synaptic GAL2 receptors on primary afferents; or anti-nociceptive effects via stimulation of
GAL1 receptors on dorsal horn neurones. ${ }^{38}$ In Coronel et al's study it is likely that the grafted MSCs exerted anti-nociceptive effects by either preventing injury-induced galanin upregulation with stimulation of pre-synaptic GAL2 receptors on primary afferents, or alternatively, stimulating GAL1 receptors. Other evidence supportive of the reparative immunological mechanisms in Franchi et al's study have shown that: the efficacy of transplanted NSCs in the treatment of neuropathic pain is mediated via neuroprotective and immunomodulatory mechanisms; ${ }^{39}$ and that in a mouse model of spared nerve injury, intra-ventricular injection of human MSCs decreased mRNA levels of the proinflammatory IL-1 gene and suppressed activation of astrocytes and microglia, which was associated with a reduction in painlike behaviours. ${ }^{40}$

The utility of intravenous systemic NSC administration in the treatment of neuropathic pain is further corroborated by evidence on the physiological mechanisms underlying NSC migration, namely, that NSCs cross the blood-brain barrier and enter the CNS where they modulate pain. ${ }^{41}$ Leading on from this, the intravenous route of NSC administration has a more transferable putative clinical application relative to other invasive methods of delivery such as intrathecal or intraventricular injection.

However, Franchi et al's results are hindered by a number of limitations. For example, other studies have found contradictory results, namely, that NSC transplantation does not affect neuropathic pain ${ }^{42}$ and may somewhat paradoxically exacerbate nociceptive symptoms. ${ }^{43}$ Furthermore, the analgesic effects were only demonstrated acutely for 28 days post-grafting, which provides no information on: the utility of NSCs in the treatment of chronic neuropathic pain; whether tolerance developed; and whether the experimental models showed delayed anti-allodynic effects. Leading on from this, during the study period repeated NSC injections were required to sustain the analgesic effect, which could be a potential hindrance to putative clinical translation in relation to patient compliance with a multi-dose regime.

The proinflammatory milieu associated with neuropathic pain may not be entirely harmful; in Franchi et al's study, anti-nociceptive effects on pain-like behaviour were observed 3 days postNSC grafting. This coincides with the time to recruit macrophages to the injury site to phagocytose myelin debris, which would otherwise have contributed to neuropathic pain.

Franchi et al reported that NSCs specifically homed towards neuronal lesions. However, this has not been supported by other studies on the biodistribution of NSCs following intravenous administration. ${ }^{44}$

Finally, Franchi et al's study results are restricted to the chronic constriction injury model of peripheral neuropathic pain and cannot be extrapolated to other neuropathic pain models.

In a separate study, NSCs were found to reduce allodynia in a central neuropathic pain model of spinal cord injury if they preferentially differentiated into oligodendrocytes rather than astrocytes ${ }^{45}$ Normally when NSCs are transplanted into the brain or spinal cord they tend to differentiate into astrocytes. ${ }^{46} \mathrm{How}$ ever, NSCs derived from the spinal cord that were virally transfected to co-express the transcription factor neurogenin-2 and 
the marker GFP, differentiated predominantly into oligodendrocytes post-transplantation. This was somewhat unexpected; in vitro neurogenin-2 normally promotes neuronal differentiation. In comparison, the naïve GFP-NSCs predictably differentiated into astrocytes. ${ }^{47}$ In a spinal cord injury model, transplanted neurogenin-2-NSCs generated more oligodendrocytes and significantly reduced allodynia relative to the naïve GFP-NSC group. The neurogenin-2-NSC grafted animals showed significantly greater white matter area relative to the naïve GFP-NSC group, which suggested the observed analgesic effects may be secondary to increased remyelination of injured axons. Interestingly, in the naïve GFP-NSC group an increased nociceptive effect was recorded. This may be explained by a higher density of naïve GFP-NSC-derived astrocytes; astrocytes in neuropathic injury models secrete trophic factors such as NGF, which promote neurite outgrowth and cell survival that facilitates locomotor and sensory recovery. ${ }^{48}$ However, the neurite outgrowth with associated nociceptive fibre sprouting into inappropriate regions of the dorsal horn may account for the pro-algesic effects observed. ${ }^{43}$

The strengths of these findings are twofold, namely: the importance of differentiating NSCs towards an oligodendrocytic lineage prior to transplantation; and specifically targeting NSC migration to precise regions of the spinal cord. This may reduce pro-nociceptive effects associated with astrocyte-derived neurotrophic factor induced neuronal sprouting into the dorsal horn.

However, in Klein et al's study no control group containing animals without neuronal lesions that received astrocyte grafts were used for comparison. This would have enabled the observers to assess whether 'healthy' control animals developed a neuropathic phenotype. Therefore, the observation that NSCderived astrocytes exacerbate neuropathic pain cannot be reliably concluded.

hMSCs have also demonstrated efficacy for the treatment of neuropathic pain. Evidence suggests hMSCs may have the best potential results for treating neuropathic pain ${ }^{49}$ and, in contrast to NSCs isolated from the SVZ, hMSCs are more readily accessible and harvested. Evidence supporting this is derived from a study that injected bone marrow derived hMSCs into the rodent tail vein of the spared nerve injury model four days after sciatic nerve surgery by which time neuropathic pain was firmly established. ${ }^{50}$

The strength of Siniscalco et al's results is the novel observation that hMSCs attenuate neuropathic pain through an anti-inflammatory restorative mechanism based on two components, namely: a cell-to-cell contact activation mechanism - hMSCs drive macrophages towards an anti-inflammatory neuroprotective M2 phenotype; and through down-regulation of proinflammatory cytokines. These findings are supported by other studies. ${ }^{51}$

No safety concerns were associated with the use of hMSCs. This coupled with the non-invasive intravenous route of administration provides for potentially favourable clinical translation. Furthermore, the inherently strong anti-inflammatory immunomodulatory properties of hMSCs, which would negate the need for pharmacological immunosuppression, adds weight to their clinical appeal. However, somewhat paradoxically, exploiting proinflammatory chemotaxis may also facilitate clinical translation; the chemokine driven homing potential of hMSCs towards pro-nociceptive lesions could be exploited as a bioactive site-specific delivery system, which would avoid the dose-limiting adverse effects associated with systemic administration of current licensed drugs.

However, the effects of hMSCs in Siniscalco et al's study are limited in their application by methodological flaws. For example, during the progression of neuropathic pain, time-course tracking of intravenous hMSCs was not performed, thus homing of hMSCs towards areas involved in neuropathic pain modulation cannot be reliably elucidated. Leading on from this, there is contradictory evidence on the homing capabilities of hMSCs towards sites of neuropathy, for example, evidence has shown that hMSCs transplanted into the mouse tail vein are predominantly sequestrated in the lung. ${ }^{52}$ Furthermore, the utility of hMSCs for the treatment of neuropathic pain was only assessed for 90 days, thus providing no information in relation to attenuating chronic neuropathic pain or reducing associated complications such as deconditioning e.g. reduced mobility, muscle atrophy and contractures.

In summary, there is an emerging body of preclinical data based on exploiting the multipotency, self-renewing capacity, high expansion potential and genetic stability of adult stem cells that supports their utility for the treatment of neuropathic pain. The mainstay of evidence has honed in on the immunomodulatory and trophic effects of adult stem cells. The progression onto clinical trials would be the next stage in defining the potential utility of adult stem cells for the treatment of neuropathic pain.

\section{Evidence on the utility of iPS cells for the treatment of neuropathic pain}

There are no published data on the utility of iPS cells for the treatment of neuropathic pain; however, there has been a lot of interest in the potential utility of iPS cells in this regard. Potential uses of iPS cells include: modelling neuropathic disease processes in vitro; developing and screening candidate drugs that selectively target diseased neuronal cells with particular genetic profiles; and offering a novel paradigm of cell replacement therapy to support neuronal regeneration.

The enormous potential utility of iPS cells for the treatment of neuropathic pain is in its infancy and remains unsupported by an evidence base. In addition to the limitations of iPS cells already mentioned, a number of other problems would need to be surmounted. For example, the retention of epigenetic profiles from senescent cells may cause iPS cells, used for neuropathic pain disease modelling or therapy, to lose their differentiated properties.

\section{Conclusions}

The evidence on the potential utility of cell therapies for the treatment of neuropathic pain is predominantly based on research in animal models on their efficacy and safety. The evidence suggests that prima facie cell therapies reduce neuropathic pain and may 


\section{Key messages}

- Cell therapies may offer palliative and curative potential in diabetic neuropathy

- Stem cell treatments for neuropathic pain reverse and repair the pathology that underlies the genesis and propagation of damage within the somatosensory system

- Stem cell therapies can replace damaged neuronal tissue, protect against progressive nerve damage, and release soluble factors to facilitate neuronal repair

modify some of the cellular and molecular neuropathic pain mechanisms. However, critical appraisal of the evidence thus far reveals it to be far from conclusive and future research geared towards progression onto clinical trials would need to address a number of issues. Firstly, the preclinical evidence reported to date suggests that in vivo cell therapies have a relatively short survival, which limits their clinical utility in the treatment of chronic neuropathic pain. In this regard future research on long-term graft viability is required. Furthermore, prior to grafting, stem cells require expansion in vitro and with increasing passaging time the stability of the cells changes, which decreases the probability of them differentiating into neurons. ${ }^{53}$ Accordingly, future research on the stability of cell therapies intended for transplantation is required. The need for future research on the issue of long-term stability and safety of cell therapy is brought into even sharper focus by the observation that following transplantation stem cell-derived grafts maintain a high proliferative potential, which carries a significant oncogenic risk. ${ }^{54}$ This was highlighted by the first case report of a donor-derived brain tumour following NSC transplantation. ${ }^{55}$

The evidence thus far on the potential disease modifying regenerative effects of cell therapies for the treatment of neuropathic pain is limited to neuropathic conditions characterised by focal nerve damage. Indeed, the mainstay of preclinical evidence has used experimental animal models with limited focal nerve damage. Future research would need to assess the potential utility of cell therapies for more diffuse and widespread nerve damage, for example in chemotherapy-induced polyneuropathy or diabetic neuropathy, which are more common than focal neuropathies.

There may be a fourth dimension on the potential utility of cell therapies for the treatment of neuropathic pain based on stem cell-derived microvesicles. Research on the utility of stem cell-derived microvesicles that carry miRNA, chemoattractant-, anti-apoptotic, and anti-scarring factors are under investigation and early results have demonstrated non-inferiority relative to cell therapies. ${ }^{56}$ However, the evidence on stem cell-derived microvesicles is sparse; future research on the role of stem cellderived microvesicles is required.

In summary, cell therapies offer a novel curative therapeutic dimension for the treatment of neuropathic pain. This is based on replacing damaged neuronal tissue, protecting against progressive nerve damage, and releasing paracrine and endocrine factors, which repair the pathology that underlies the genesis and propagation of damage within the somatosensory system.

\section{Conflict of interest None Funding sources None}

\section{References}

1. Ziegler D, Rathmann W, Dickhaus T et al. for the KORA Study Group. Prevalence of polyneuropathy in prediabetes and diabetes is associated with abdominal obesity and microangiopathy: the MONICA / KORA Augsbury Surveys S2 and S3. Diabetes Care 2008;31:464-9. http://dx.doi.org/10.2337/dc07-1796

2. Freynhagen R, Bennett Ml. Diagnosis and management of neuropathic pain. BMJ 2009;339:391-5. http://dx.doi.org/10.1136/bmj.b3002

3. Scholz J, Woolf CJ. The neuropathic pain triad: neurons, immune cells and glia. Nat Neurosci 2007;10:1361-8. http://dx.doi.org/10.1038/nn1992

4. Burnett MG, Zager EL. Pathophysiology of peripheral nerve injury: a brief review. Neurosurg Focus 2004;16:E1. http://dx.doi.org/10.3171/foc.2004.16.5.2

5. Dworkin $\mathrm{RH}, \mathrm{O}^{\prime}$ Connor $\mathrm{AB}$, Audette J et al. Recommendations for the pharmacological management of neuropathic pain: an overview and literature update. Mayo Clin Proc 2010;85:Suppl 3,S3-14. http://dx.doi.org/10.4065/mcp.2009.0649

6. Finnerup NB, Otto M, McQuay HJ et al. Algorithm for neuropathic pain treatment: an evidence based proposal. Pain 2005;118:289-305. http://dx.doi.org/10.1016/j.pain.2005.08.013

7. Mallis A, Furlan A. Sympathectomy for neuropathic pain. Cochrane Database Syst Rev 2003; 2: CD002918.

8. Pittler MH, Ernst E. Complementary therapies for neuropathic and neuralgic pain: systematic review. Clin J Pain 2008;24:731-3. http://dx.doi.org/10.1097/AJP.0b013e3181759231

9. Thompson JA, Itskovitz-Eldor J, Shapiro SS et al. Embryonic stem cell lines derived from human blastocysts. Science 1998;282:1145-7. http://dx.doi.org/10.1126/science.282.5391.1145

10. Gershon D. Complex political, ethical and legal issues surround research on human embryonic stem cells. Nature 2003;422:928-9. http://dx.doi.org/10.1038/nj6934-928a

11. Muttini A, Valbonetti $L$, Abate $M$, et al. Ovine amniotic epithelial cells: In vitro characterization and transplantation into equine superficial digital flexor tendon spontaneous defects. Res Vet Sci 2013;94:158-69. http://dx.doi.org/10.1016/j.rvsc.2012.07.028

12. Díaz-Pado S, Muiños-López E, Hermida-Gómez T, et al. Human amniotic membrane as an alternative source of stem cells for regenerative medicine. Differentiation 2011;81:162-71. http://dx.doi.org/10.1016/j.diff.2011.01.005

13. Ralston $A$, Rossant J. The genetics of induced pluripotency. Reproduction 2012;139:35-44. http://dx.doi.org/10.1530/REP-09-0024

14. Takahashi K, Yamanaka S. Induction of Pluripotent Stem Cells from Mouse Embryonic and Adult Fibroblast Cultures by Defined Factors. Cell 2006;126:663-76. http://dx.doi.org/10.1016/j.cell.2006.07.024

15. Mallanna SK, Rizzino A. Emerging roles of microRNAs in the control of embryonic stem cells and the generation of induced pluripotent stem cells. Dev Biol 2010;344:16-25. http://dx.doi.org/10.1016/j.ydbio.2010.05.014

16. Dohoon K, Chun-Hyung K, Jung-II M et al. Generation of Human Induced Pluripotent Stem Cells by Direct Delivery of Reprogramming Proteins. Cell Stem Cell 2009;4:472-76. http://dx.doi.org/10.1016/j.stem.2009.05.005

17. Stadtfeld M, Nagaya M, Utikal J et al. Induced pluripotent stem cells generated without viral integration. Science 2008;322:945-49. http://dx.doi.org/10.1126/science.1162494

18. Takahashi K, Tanabe K, Ohnuki M et al. Induction of pluripotent stem 
cells from adult fibroblasts by defined factors. Cell 2007;131:861-72. http://dx.doi.org/10.1016/j.cell.2007.11.019

19. Klusakova I, Dubovy P. Experimental models of peripheral neuropathic pain based on traumatic nerve injuries - an anatomical perspective. Ann Anat 2009;191:248-59. http://dx.doi.org/10.1016/j.aanat.2009. 02.007

20. Odd-Gehir B. Predictive validity of behavioural animal models for chronic pain. Br J Pharmacol 2011;164:1195-206. http://dx.doi.org/10.1111/j.1476-5381.2011.01300.x

21. Le Bars D., Gozariu M, Cadden SW et al. Animal models of nociception. Pharmacol Rev 2001:53:597-652.

22. Kawasaki-Yatsugi S, Nagakura Y, Ogino S et al. Automated measurement of spontaneous pain-associated limb movement and drug efficacy evaluation in a rat model of neuropathic pain. Eur J Pain 2012;16:142636. http://dx.doi.org/10.1002/j.1532-2149.2012.00142.x

23. Braz JM, Sharif-Naeini R, Vogt $D$ et al. Forebrain GABAergic neuron precursors integrate into adult spinal cord and reduce injury-induced neuropathic pain. Neuron 2012;74:663-75.

http://dx.doi.org/10.1016/j.neuron.2012.02.033

24. Vit JP, Ohara PT, Sundberg $C$ et al. Adenovector GAD65 gene delivery into the rat trigeminal ganglion produces orofacial analgesia. Mol Pain 2009:5:42.

25. Hao S, Mata M, Wolfe $D$ et al. Gene transfer of glutamic acid decarboxylase reduces neuropathic pain. Ann Neurol 2005;57:914-18. http://dx.doi.org/10.1002/ana.20483

26. Eaton MJ, Plunkett JA, Martinez MA et al. Transplants of neuronal cells bioengineered to synthesize GABA alleviate chronic neuropathic pain. Cell Transplant 1999:8:87-101.

27. Mackie M, Hughes DI, Maxwell DJ et al. Distribution and colocalisation of glutamate decarboxylase isoforms in the rat spinal cord. Neuroscience 2003;119:461-72. http://dx.doi.org/10.1016/S0306-4522(03)00174-X

28. De Koninck Y. Altered chloride homeostasis in neurological disorders: a new target. Curr Opin Pharmacol 2007;7:93-9. http://dx.doi.org/10.1016/j.coph.2006.11.005

29. Price TJ, Cervero F, de Konnick Y. Chloride regulation in the pain pathway. Brain Res 2009;60:149-70.

30. Hendricks WA, Pak ES, Owensby JP et al. Predifferentiated embryonic stem cells prevent chronic pain behaviours and restore sensory function following spinal cord injury in mice. Mol Med 2006;12:34-46. http://dx.doi.org/10.2119/2006-00014. Hendricks

31. Hains BC, Johnson KM, Hulseboch CE. Engraftment of serotonergic precursors enhances locomotor function and attenuates chronic central pain behaviour following spinal hemisection injury in the rat. Exp Neurol 2001;171:361-78. http://dx.doi.org/10.1006/exnr.2001.7751

32. Franchi S, Valsecchi A, Borsani E et al. Intravenous neural stem cells abolish nociceptive hypersensitivity and trigger nerve regeneration in experimental neuropathy. Pain 2012;153(4):850-61. http://dx.doi.org/10.1016/ j.pain.2012.01.008

33. Martucci C, Trovato AE, Costa B et al. The purinergic antagonist PPADS reduces pain related behaviours and interleukin-1 beta, interleukin-6, iNOS and nNOS overproduction in central and peripheral nervous system after peripheral neuropathy in mice. Pain 2008;137:81-95. http://dx.doi.org/10.1016/j.pain.2007.08.017

34. Ruff CA, Wilcox JT, Fehlings MG. Cell-based transplantation strategies to promote plasticity following spinal cord injury. Exp Neurol 2012;235:78-90. http://dx.doi.org/10.1016/j.expneurol.2011. 02.010

35. Harris JA. Using c-fos as a neural marker of pain. Brain Res Bull 1998;45:1-8. http://dx.doi.org/10.1016/S0361-9230(97)00277-3

36. Cahill CM, Coderre TJ. Attenuation of hyperalgesia in a rat model of neuropathic pain after intrathecal pre- or post-treatment with a neurokinin-1 antagonist. Pain 2002;95:277-85. http://dx.doi.org/10.1016/\$0304-3959(01)00410-9

37. Coronel MF, Musolino PL, Brumovsky PR et al. Bone marrow stromal cells attenuate injury-induced changes in galanin, NPY and NPY Y1-receptor expression after a sciatic nerve constriction. Neuropeptides 2009;43:12532. http://dx.doi.org/10.1016/i.npep.2008.12.003

38. Liu HX, Hokfelt T. The participation in galanin in pain processing at the spinal level. Trends Pharamacol Sci 2002:23:468-74 http://dx.doi.org/10.1016/S0165-6147(02)02074-6
39. Pluchino S, Gritti A, Blezer E et al. Human neural stem cells ameliorate autoimmune encephalomyelitis in non-human primates. Ann Neurol 2009;66:343-54. http://dx.doi.org/10.1002/ana.21745

40. Siniscalco D, Giordano C, Galderisi U et al. Intra-brain microinjection of human mesenchymal stem cells decreases allodynia in neuropathic mice. Cell Mol Life Sci 2012:67:655-69. http://dx.doi.org/10.1007/s00018-009-0202-4

41. Bacigaluppi M, Pluchino S, Peruzzotti-Jametti $L$ et al. Delayed post-ischaemic neuroprotection following neural stem cell transplantation involves multiple mechanisms. Brain 2009;132:2239-51. http://dx.doi.org/10.1093/brain/awp174

42. Karimi-Abdolrezaee S, Eftekharpour E, Wang J et al. Synergistic effects of transplanted adult neural stem / progenitor cells, chondroitinase, and growth factors promote functional repair and plasticity of chronically injured spinal cord. J Neurosci 2010;30:1657-76.

http://dx.doi.org/10.1523/JNEUROSCI.3111-09.2010

43. Hofstetter CP, Holmstrom N, Lilja JA et al. Allodynia limits the usefulness of intraspinal neural stem cell grafts; directed differentiation improves outcome. Nat Neurosci 2005:8:346-53. http://dx.doi.org/10.1038/nn1405

44. Pendharkar AV, Chua JY, Andres RH et al. Biodistribution of neural stem cells after intravascular therapy for hypoxic-ischaemia. Stroke 2010;41 2064-70. http://dx. doi.org/10.1161/STROKEAHA.109.575993

45. Klein S, Svendsen CN. Stem cells in the injured spinal cord: reducing the pain and increasing the gain. Nature Neurosci 2005;8:259-60. http://dx.doi.org/10.1038/nn0305-259

46. Winkler RA, Fricker MA, Gates MA et al. Incorporation and glial differentiation of mouse EGF-responsive neural progenitor cells after transplantation into the embryonic rat brain. Mol Cell Neurosci 1998;11 99-116.

47. Sommer L, Ma Q, Anderson DJ. Neurogenins, a novel family of atonalrelated $\mathrm{bH} \mathrm{LH}$ transcription factors, are putative mammalian neuronal determination genes that reveal progenitor cell heterogeneity in the developing CNS and PNS. Mol Cell Neurosci 1996:8:221-41. http://dx.doi.org/10.1006/mcne.1996.0060

48. Brown A, Ricci MJ, Weaver LC. NGF message and protein distribution in the injured rat spinal cord. Exp Neurol 2004;188:115-27. http://dx.doi.org/10.1016/j.expneurol.2004.03.017

49. Klass M, Gavrikov V, Drury D et al. Intravenous mononuclear marrow cells reverse neuropathic pain from experimental mononeuropathy. Anesth Analg 2007;104:944-48. http://dx.doi.org/10.1213/01.ane. 0000258021.03211.d0

50. Siniscalco D, Giordano C, Galderisi U et al. Long-lasting effects of human mesenchymal stem cell systemic administration on pain-like behaviours, cellular, and biomolecular modifications in neuropathic mice. Frontiers in Integrative Neuroscience 2011:5:1-10. http://dx.doi.org/10.3389/fnint.2011.00079

51. Kim J, Hematti P. Mesenchymal stem cell-educated macrophages: a novel type of alternatively activated macrophages. Exp Hematol 2009;37 1445-53. http://dx.doi.org/10.1016/j.exphem.2009.09.004

52. Ankrum I, Karp J. Mesenchymal stem cell therapy: two steps forward, one step back. Trends Mol Med 2010;16:203-09. http://dx.doi.org/10.1016/j.molmed.2010.02.005

53. Jain $\mathrm{M}$, Armstrong RJ, Tyers $\mathrm{P}$ et al. GABAergic immunoreactivity is predominant in neurons derived from expanded human neural precursor cells in vitro. Exp Neurol 2003:182:113-23. http://dx.doi.org/10.1016/S0014-4886(03)00055-4

54. Mukhida K, Mendez I, McLeod M et al., Spinal GABAergic transplants attenuate mechanical allodynia in a rat model of neuropathic pain. Stem Cells 2007:25:2874-85. http://dx.doi.org/10.1634/stemcells.2007-0326

55. Amariglio N, Hirshberg A, Scheithauer BW et al. 'Donor-derived brain tumor following neural stem cell transplantation in an ataxia telangiectasia patient.' PLOS Medicine 2009:17:e:1000029.

56. Herrera MB, Fonsato V, Gatti S et al. Human liver stem cell-derived microvesicles accelerate hepatic regeneration in hepatectomised rats. J Cell Mol Med 2010;14:1605-18. http://dx.doi.org/10.1111/j.1582-4934.2009.00860.x 